\title{
On the mode of operation in electrolyte-gated thin film transistors based on different substituted polythiophenes
}

\author{
Henrik Toss, Clement Suspene, Benoit Piro, Abderrahim Yassar, Xavier Crispin, Loig \\ Kergoat, Minh-Chau Pham and Magnus Berggren
}

\section{Linköping University Post Print}

\section{Tweet}

N.B.: When citing this work, cite the original article.

Original Publication:

Henrik Toss, Clement Suspene, Benoit Piro, Abderrahim Yassar, Xavier Crispin, Loig Kergoat, Minh-Chau Pham and Magnus Berggren, On the mode of operation in electrolyte-gated thin film transistors based on different substituted polythiophenes, 2014, Organic electronics, (15), $10,2420-2427$. http://dx.doi.org/10.1016/j.orgel.2014.06.017

Copyright: Elsevier http://www.elsevier.com/

Postprint available at: Linköping University Electronic Press http://urn.kb.se/resolve?urn=urn:nbn:se:liu:diva-110957 


\title{
On the mode of operation in electrolyte-gated thin film transistors based on different substituted polythiophenes
}

\author{
Henrik Toss ${ }^{(a)}$, Clément Suspène ${ }^{(b)}$, Benoît Piro ${ }^{(b)}$, Abderrahim Yassar ${ }^{(c)}$, Xavier Crispin ${ }^{(a)}$, Loïg \\ Kergoat $^{(\mathrm{a})}$, Minh-Chau Pham ${ }^{(\mathrm{b})}$, Magnus Berggren ${ }^{(\mathrm{a})^{*}}$
}

(a) Department of Science and Technology (ITN), Linköpings Universitet, Campus Norrköping, Bredgatan 34, 60174 Norrköping (Sweden)

(b) Univ. Paris Diderot, Sorbonne Paris Cité, ITODYS, UMR 7086 CNRS, 15 rue J-A de Baïf, 75205 Paris Cedex 13, France

(c) LPICM, UMR CNRS 7647, Ecole Polytechnique, Route de Saclay, 91128 Palaiseau (France)

*Corresponding Author; Email: magnus.berggren@liu.se; Phone: +46 11363637

\begin{abstract}
Organic Thin Film Transistors (OTFT), gated through an aqueous electrolyte, have extensively been studied as sensors in various applications. These water-gated devices are known to work both as electrochemical (Organic ElectroChemical Transistor - OECT) and field-effect (Organic Field-Effect Transistor - OFET) devices. To properly model and predict the response of water-gated OTFT sensors it is important to distinguish between the mechanism, field-effect or electrochemical, by which the transistor is modulated and thus how the gate signal can be affected by the analyte. In this present study we explore three organic polymer semiconductors, poly-(3-hexyl-thiophene) (P3HT), poly-(3carboxy-pentyl-thiphene) (P3CPT) and a co-polymer P3HT-co-poly-(3-ethoxypentanoic acidthiophene) (monomer ratio 1:6, P3HT-COOH15) in water-gated OTFT structures. We report a set of transistor characteristics, including standard output parameters, impedance spectroscopy and current transients, to investigate the origin of the mode of operation in these water-gated OTFTs. Impedance characteristics, including both frequency and voltage dependence, were recorded for capacitor stacks corresponding to the gate/electrolyte/semiconductor/source structure. It is shown that $\mathrm{P} 3 \mathrm{HT}$ as well as P3HT-COOH15 both can function as semiconductors in water gated OTFT devices operating in field-effect mode. P3CPT on the other hand shows typical signs of electrochemical mode
\end{abstract}


of operation. The $-\mathrm{COOH}$ side group has been suggested as a possible anchoring site for biorecognition elements in EGOFET sensors, rendering P3HT-COOH15 a possible candidate for such applications.

\section{Highlights}

- When gated through an aq. media and depending on the concentration of carboxylic anchoring groups, the polythiophene material system can operate either in the pure fieldeffect or in the pure electrochemical mode of operation.

- In the polythiophene derivative with $15 \%$ of all the thiophene rings including $\mathrm{COOH}$ containing substituents (anchoring sites for receptors), the material can function as the transistor channel in a TFT operating in the field-effect mode of operation.

- However, with $\mathrm{COOH}$-groups attached to every thiophene ring the semiconductor material operates in the pure electrochemical mode when included in a TFT structure.

- Our studies includes and presents a set of spectroscopic and current vs. voltage measurements which together provide a tool for scientists to judge upon the mode of operation, field-effect or electrochemical, in organic water-gated TFTs for sensor applications.

- The polythiophene material system, including $15 \%$ of anchoring sites, is thus an excellent candidate for potentiometric applications and in sensor applications in which the receptorto-transistor transduction rely on sensor signal transfer occurring at the electrolyte-water interface.

Keywords: Sensor, OTFT, transistor, electrolyte, EGOFET

\section{Introduction}

Presently, there is a tremendous activity to explore organic semiconducting materials because they can be processed from solution at low temperature [1-8] hence, traditional printing technologies can be adapted to manufacture printed sensors and detectors. Such types of devices represent an important corner stone for applications such as distributed monitoring and medical surveillance. The printed sensors are typically comprised of bio-recognition elements and a signal 
transducer device; which could e.g. be an organic thin film transistor. The OTFTs typically run either in a field-effect (OFET) or an electrochemical (OECT) mode of operation, i.e. current modulation is due to either charge polarization occurring along the aqueous electrolyte-semiconductor interface or chemical doping occurring within the bulk of the semiconductor channel $[1-3,6,9-11]$. Organic thin film transistors (OTFT) hosting antibodies, peptides or enzymes have been developed for applications in aqueous and physiological media and have been studied extensively in the past [1-5, 7, 12-17]. The bio-recognition elements can be either attached to the surface of the gate in a top-gate configuration (Figure 1a) $[2-3,5,7,18]$; or on the surface of the semiconductor in a bottom gate structure using a solid insulator layer (Figure 1b) [19-23].

In a new version of the OTFT sensors, the electrolyte media carrying the analyte, is located between the gate and the semiconducting channel. In other words, the electrolyte serves as gate-insulator for the transistor (Figure 1c). In this case, the drain current modulation and the transistor performance rely on the electric field established across the semiconductor-aqueous media interface. For these electrolyte-gated OTFTs (EGOTFTs) a capacitance is built up as charge is injected into the organic semiconductor (OSC) at the interface, to compensate for the charges stored within the Helmholtz layer of the electrolyte. The properties of this electric double layer interface, and the corresponding capacitance, are thus crucial to the transistor characteristics. It is attractive to anchor sensor receptors directly at the OFET channel, since the receptor reaction can cause modulation of the trap density, morphology, dipoles etc, thus causing control of the drain current. Assuming that the bio-recognition element is located on the surface of the semiconductor, upon reaction with the analyte, the charge capacity and dynamics of the Helmholtz double-layer is expected to be modified; which should results in a variation of the static and/or dynamic transistor responses. The speed of EGOFETs gated via water is primarily dictated by the ion diffusion rate and concentration of the aqueous electrolyte. For OECTs the speed of operation is typically governed by ion migration within the bulk semiconductor and thus appears relatively slower than the EGOFETs [11]. One strategy to immobilize the bio-recognition element along the surface of the OSC channel 
surface is to utilize molecular anchors, such as carboxyl groups [24-25]. Similar methods have been applied in EGOTFT-sensors in the past. In those cases the anchoring sites have either not been attached directly to the semiconductor or the mechanism of transistor actuation has been unclear. The inclusion of anchoring sites on the semiconducting polymer may affect the mode of operation of the OTFT [11]. Previous studies on solid electrolyte gated OTFTs, i.e. using architectures not appropriate for sensors, indicate that the chemical nature of the semiconducting polymers is crucial since it affects the operation mechanisms. The EGOTFTs typically run either in a field-effect (OFET) [26]or an electrochemical (OECT) mode of operation P3CPT, [11], i.e. current modulation is due either to charge polarization occurring along the aqueous analyte-semiconductor interface or chemical doping occurring within the bulk of the semiconductor channel.

Here, we investigate the impact of the bio-recognition anchoring groups on the mode of operation of EGOTFTs for bio-sensor applications. Three different polythiophene derivatives with various ratio of anchoring groups are compared: poly-(3-hexyl-thiophene) P3HT (Figure 2a), poly-(3carboxy-pentyl-thiphene) P3CPT (Figure 2b) and a random co-polymer P3HT-co-Poly-(3ethoxypentanoic acid-thiophene) (monomer ratio 1:6, i.e. 15\% - $\mathrm{COOH}$ side chain end groups, P3HT$\mathrm{COOH} 15$ [27-28]) (Figure 2c) are compared.

\section{Experimental}

\subsection{Production of devices}

The capacitor and transistor devices are fabricated according to the same process protocol; the only difference is the bottom metal electrode pattern. The top electrode of the capacitor stack is identical to the gate electrode of the transistors. Capacitor and transistor devices (see Figure 3) are fabricated on Si-wafers with a thick (10 $000 \AA$ A) thermally grown oxide layer on top. As the first process step, a thin attachment layer of Ti (50 $⿱$ ) is vacuum evaporated on the oxide surface. Then, a $500 \AA$ layer of $\mathrm{Au}$ is vacuum evaporated on top of the Ti attachment layer. The 
patterned Au electrodes are then generated using standard UV-photolithography (Suss MA/BA 6 mask and bond aligner and soda lime glass masks with patterned chromium from Delta Mask) followed by chemical wet etching $\left(1 / \mathrm{KI}\right.$ in $\mathrm{H}_{2} \mathrm{O}$ for $\mathrm{Au}$ and $\mathrm{NH}_{3} / \mathrm{H}_{2} \mathrm{O}_{2}$ in $\mathrm{H}_{2} \mathrm{O}$ for Ti etch). Electrode patterns for capacitors and transistors are generated, $1 * 1 \mathrm{~mm}^{2}$ patterns for the capacitors and interdigitated electrodes with channel length $(L)$ of $2 \mu \mathrm{m}$ and width $(W)$ of 2-10 $\mathrm{mm}$ for the transistors. P3HT is purchased from Sigma (electronic grade, $15000-45000 \mathrm{u}$ ) and P3CPT is purchased from Rieke (Item \#4032, 55000-65000 u). The third polymer, P3HT-COOH15, is synthesized and provided by Université Paris Diderot ( 18000 u random co-polymer)[27] Solutions of the polymers are prepared by dissolving $\mathrm{P} 3 \mathrm{HT}$ in 1,2-dichlorobenzene (ODCB) at a concentration of $10 \mathrm{mg} / \mathrm{ml}$, P3CPT in dimethyl sulfoxide (DMSO) at a concentration of $10 \mathrm{mg} / \mathrm{ml}$ and the co-polymer, P3HT$\mathrm{COOH} 15$, is dissolved in $\mathrm{ODCB}$ at a concentration of approximately $10 \mathrm{mg} / \mathrm{ml}$. The polymers are deposited on the bottom-electrode substrates by spin coating the solutions at $3000 \mathrm{rpm}$ for $90 \mathrm{~s}$, in ambient atmosphere, with the substrates heated to $150{ }^{\circ} \mathrm{C}$. After spin coating, the samples are vacuum-baked at $120{ }^{\circ} \mathrm{C}$ for $120 \mathrm{~s}$ followed by an annealing step at $120{ }^{\circ} \mathrm{C}$ in $\mathrm{N}_{2}$ for $20 \mathrm{~min}$. A $7 \mu \mathrm{L}$ droplet of deionized water, enough to cover the entire channel and the interdigitated bottom electrodes, is applied directly on top of the semiconductor surface. The Au-drain and -source electrodes extend into contact pads making electrical probing possible away from the water droplet. Finally, a Au-foil stripe is applied to the top of the water droplet to serve as the gate. The gate stripe is immersed into the water droplet to make sure that the gate area, i.e. the contact area between the gate and the water droplet, is around 4 times larger than the area of the bottom drain and source electrodes. This configuration guarantees that the limiting capacitor is located at the water-OSC interface.

\subsection{Capacitor characterization}

The frequency dependence of the impedance of the capacitance stack is investigated using a Novocontrol Alpha High Resolution Dielectric Analyzer. An AC signal of constant amplitude is 
applied and the impedance is recorded as the frequency of the applied signal is scanned from $1 \mathrm{MHz}$ down to $100 \mathrm{mHz}$.

The capacitors, including the three polythiophenes, are also evaluated in capacitance vs. voltage (CV) measurements. This measurement is carried out by sweeping a DC offset voltage (0.7 to $1.7 \mathrm{~V})$ combined with applying an $\mathrm{AC}$ voltage $\left(V_{P P}=0.1 \mathrm{~V}, f=100 \mathrm{~Hz}\right)$ to the two electrodes of the capacitors.

\subsection{Transistor output characteristics}

The output characteristics of the transistors are recorded using a Kiethley 4200 Semiconductor Characterization System. This is done for six different, constant gate-source voltages ranging from 0 to $-0.5 \mathrm{~V}$ while sweeping the drain-source voltage from 0 to $-0.5 \mathrm{~V}$.

\subsubsection{Characterization of the response speed in transistor devices through observation of transient behavior.}

The switching time of the transistors is estimated by recording the transient response of the transistors when a step function of the gate voltage is applied. During this test the drainsource voltage is kept constant at $-0.5 \mathrm{~V}$. A $0 \mathrm{~V}$ gate-source voltage is initially applied and, in a step function, changed to $-0.5 \mathrm{~V}$ and kept constant at this voltage for approximately $60 \mathrm{~s}$, after which it is switched back, in one step, to $0 \mathrm{~V}$. The transistor Drain (and Gate) current(s) is recorded as a function of time to provide information about the OTFT transient behaviour.

\section{Results}

\subsection{Capacitor characterization}


Capacitor structures are fabricated with a $1 \mathrm{~mm}^{2}$ bottom $\mathrm{Au}$ electrode and are manufactured on the Si-wafer onto which the semiconductor is coated so that it covers the entire area of the bottom electrode. From ellipsometry, we find that the thicknesses of the semiconductor layers are $20 \mathrm{~nm}$ (P3HT) and $10 \mathrm{~nm}$ (P3CPT, P3HT-COOH15). A water droplet, large enough to overlap the entire bottom electrode area (approximately $7 \mu \mathrm{L}$ ), is then dispensed on top of the semiconductor surface. Finally, a flat Au foil, held in place by a probe holder, with the approximate dimension of 3*2 $\mathrm{mm}$ is immersed into the water droplet so that the electrode surface is in electrical contact with the droplet but not in direct contact with the semiconductor nor the bottom electrode. Impedance characteristics for capacitor structures, including the three OSC, corresponding to the gate-water-OSC-source configuration is given in Figure 4. We interpret and model the impedance parameters using a serial, resistor-capacitor model. This model represents the two interface capacitances (Helmholtz layers) and the bulk resistors (water and the OSC). The resistor and capacitor are primarily represented by the ionic conduction of DI water (largest resistor) and the EDL at the water-OSC interface (smallest capacitor), respectively. Assuming none or very low rate of kinetics we exclude Warburg impedance contribution. At high $(1 \mathrm{MHz})$ and at low $(1 \mathrm{~Hz})$ frequencies the capacitors including P3HT shows a phase angle close to $\varphi=-90^{\circ}$ corresponding to a more or less pure capacitive behavior. In the high frequency region, the impedance characteristics in the capacitor is dominated by the dipolar relaxation of the water, then function as an insulator, and is mimicking a dielectric behavior $\left(C_{i}=1 \mathrm{nF} / \mathrm{cm}^{2}\right)$. At low frequencies, dissociated $\mathrm{OH}^{-}$and $\mathrm{H}_{3} \mathrm{O}^{+}$has enough time to migrate inside the water droplet to build up dense Helmholtz layers along the semiconductor and $\mathrm{Au}$ electrode surface, respectively. The highest occupied molecular orbital (HOMO) of the three polythiophenes are located in the range from approximately -4.8 to $-5.11 \mathrm{eV}$ [29-32] (the HOMO level for P3HT-COOH15 has been determined by UV-visible spectroscopy and by cyclic voltammetry at the Université Paris Diderot to be $-5.11 \mathrm{eV})$. The work function of Au is typically found in the range from 5.1 to $5.3 \mathrm{eV}$ [33]. This means that the Au electrode forms an ohmic contact to all the three OSCs. So, at low voltages, and since $\mathrm{Au}$ is also used as the gate electrode, we can neglect any impedance 
contribution from the OCSs, since holes can easily migrate in and out from the bulk of the thin OSC layer. This means that dense Helmholtz layers are formed at the OSC-water and at the water-Au interfaces. The calculated effective capacitance of the stack including P3HT is on the order of the value of a typical Helmholtz layer measured in planar water-electrode configurations, i.e. approximately $10 \mu \mathrm{F} / \mathrm{cm}^{2}$ [26], see Figure 4 . In the intermediate frequency region, i.e. from $100 \mathrm{~Hz}$ to $100 \mathrm{kHz}$, the impedance characteristics are primarily dominated by $\mathrm{OH}^{-}$and $\mathrm{H}_{3} \mathrm{O}^{+}$relaxation and transport. This then represents the region where the capacitor incrementally transforms between a dielectric and an electric double layer type of capacitance behavior. In this intermediate region the phase angle is close to $\varphi=0^{\circ}$ and, thus, the device exhibits a purely resistive behavior. P3HT-COOH15 shows a similar behavior, as compared to $\mathrm{P} 3 \mathrm{HT}$, however, the phase angle reaches only about $\varphi=-$ $80^{\circ}$ for the low $(1 \mathrm{~Hz})$ and high $(1 \mathrm{MHz})$ frequency regions. The calculated effective capacitance $\left(C_{i}\right)$ for P3HT-COOH15, at low frequencies, is however also on the same order of magnitude as what can be expected for a Helmholtz layer (double layer capacitance). P3CPT has yet an even less negative phase angle value for the low frequency region, $\varphi=-70^{\circ}$. Moreover, the effective capacitance is here about one order of magnitude higher than what has been observed for a similar system with a bottom electrode of Au alone, $\sim 10-100 \mu \mathrm{F} / \mathrm{cm}^{2}[26,34]$, and, thus, what could be expected from a double layer capacitance. This indicates that there is another mechanism that further boosts the capacitance value at the low frequency region for P3CPT. Reduction and oxidation of conjugated polymer thin (10-100 nm) films typically results in a charge capacity values equivalent to an effective capacitance on the order of $100 \mu \mathrm{F} / \mathrm{cm}^{2}$.

The capacitors, including the three polythiophenes, are also evaluated in capacitance vs. voltage (CV) measurements. This measurement is carried out by sweeping a DC offset voltage (0.7 to $1.7 \mathrm{~V})$ combined with applying an $A C$ voltage $\left(V_{P P}=0.1 \mathrm{~V}\right)$ to the two electrodes of the capacitors. The CV characteristics can provide crucial information about polarization and storage of 
charges as a function of the magnitude and polarity of the electric field over the capacitor stack. For P3HT and P3HT-COOH15 we find a typical transition from a low $-C_{i}\left(\sim 0.1 \mu \mathrm{F} / \mathrm{cm}^{2}\right.$ for P3HT and 0.3 $\mu \mathrm{F} / \mathrm{cm}^{2}$ for $\left.\mathrm{P} 3 \mathrm{HT}-\mathrm{COOH} 15\right)$ to a high- $\mathrm{C}_{i}$ regime $\left(\sim 3 \mu \mathrm{F} / \mathrm{cm}^{2}\right.$ for $\mathrm{P} 3 \mathrm{HT}$ and $\left.\mathrm{P} 3 \mathrm{HT}-\mathrm{COOH} 15\right)$, see Figure 5. All three polymers show a plateau at high positive voltages. At high positive voltages, no charges are injected into the OSCS and the polythiophene films then act as dielectric layers. Below a certain voltage, that is applied to the top electrode, holes are injected into the OSCS and a Helmholtz layer can now be formed along the water-OSC interface. The formation of this Helmholtz layer should ideally form yet another plateau in the $\mathrm{C}-\mathrm{V}$ in the low-voltage regime. This seems valid for P3HT$\mathrm{COOH} 15$ and $\mathrm{P} 3 \mathrm{HT}$, at least down to $-0.4 \mathrm{~V}$. However, the incremental step in the CV characteristics differs by around $0.4 \mathrm{~V}$ between the two materials. We address this difference in the C-V step to a difference in the HOMO level between the two polymers (4.8 eV for P3HT and $5.11 \mathrm{eV}$ for P3HT$\mathrm{COOH} 15)$ and also that the $\mathrm{COOH}$-groups, in $\mathrm{P} 3 \mathrm{HT}-\mathrm{COOH} 15$, may introduce dipoles along the semiconductor-electrolyte interface. For P3CPT no clear saturation of the $C_{i}$ characteristics is found in the low-voltage region, what so ever. Instead, the capacitance value keeps increasing, even as the voltage turns negative. Also, the value of the capacitance approaches $10^{-4} \mathrm{~F} / \mathrm{cm}^{2}$ at bias levels below $0 \mathrm{~V}$. This means that the charge capacity continuously increases, i.e. the holes that are injected from the bottom electrode into the bulk of P3CPT becomes compensated at a level beyond what can be stored along a Helmholtz layer.

\subsection{Transistor output characteristics}

Transistor structures (Figure $3 \mathrm{~b}$ ) are identical to the capacitor structures reported above, with the only exception that the bottom electrodes are represented by interdigitated bottom Au source and drain electrodes corresponding to a channel length and width of $2 \mu \mathrm{m}$ and 2-10 mm, respectively. Also, the Au foil, serving as the gate, is here slightly smaller $(1 * 2 \mathrm{~mm})$ 
The transistor I-V characteristics were recorded by sweeping the drain-source voltage at a $170 \mathrm{mV} / \mathrm{s}$ rate and the gate-source voltage was incrementally $(0.1 \mathrm{~V})$ switched from 0 to $-0.5 \mathrm{~V}$. For the different sets of IV curves (Figure 6) a clear saturation in the $I_{D}$ Vs. $V_{D S}$ curves can be observed for P3HT (a) and P3HT-COOH15 (b) with $I_{D}$ levels peaking at 3 to $4 \mu \mathrm{A} \cdot \mathrm{mm}^{-1}$, at $V_{G S}=-0.5 \mathrm{~V}$. In the case of P3CPT no clear ID saturation is found and the drain current level that reaches beyond $100 \mu A \cdot m^{-1}$ at $V_{G S}=-0.5 \mathrm{~V}$. There is a small $\mathrm{I}_{\mathrm{D}}$ hysteresis for $\mathrm{P} 3 \mathrm{HT}$ as well as for $\mathrm{P} 3 \mathrm{HT}-\mathrm{COOH} 15$, with the reverse sweep current always being slightly lower than the forward one. This effect could be a result of bias stress, which for p-type transistors normally shifts the threshold voltage towards a more negative value [35]. For P3CPT, on the other hand, the current of the reverse sweep is relatively much larger as compared to the forward sweep. This indicates that accumulation of charges takes a long time to establish within and along the channel [11]. A drain current level of 1 to $10 \mu \mathrm{A} . \mathrm{mm}^{-1}$ is typical for a field-effect transistor, of similar dimensions as used here, which operates in the on-state and that includes a semiconductor with a mobility ranging from 0.01 to $0.1 \mathrm{~cm}^{2} / \mathrm{Vs}$.

\subsubsection{Characterization of the response speed in transistor devices through observation of transient behavior.}

The transistor transient behavior (on/off switching) was examined by applying a step voltage to the gate electrode while recording the drain and gate currents as a function of time. $\mathrm{P} 3 \mathrm{HT}$ and $\mathrm{P} 3 \mathrm{HT}$ $\mathrm{COOH} 15$ both exhibits a relatively fast turn on/off response as the gate is switched between 0 and $0.5 \mathrm{~V}$ (Figure 7a,b). Especially, the off current level is swiftly reached as the transistor is turned off $\left(V_{G S}\right.$ is switched from $-0.5 \mathrm{~V}$ to $\left.0 \mathrm{~V}\right)$. The turn-on transient includes an initial relatively fast component and a second slower one. The quick turn-on component reflects the formation of the Helmholtz layer and the associated establishment of the 2D transistor channel. The slow component could possibly be due to rearrangement of the conjugated polymer chains along the interface or a shift of the threshold voltage due to bias stress/trap filling. This slow turn-on component is more pronounced for P3HT-COOH15 as compared to P3HT. Transistors including P3CPT as the semiconductor also show one fast and one slow IDs transient component upon turning on the transistor (Figure 7c). However, 
in this case the slow turn-on component completely dominates the overall drain current level and evolution. Upon turning off P3CPT-transistors the off currents do not return immediately to their steady-state off-current level. In fact, this is only achieved after an extensive period of time, i.e. typically after $>20 \mathrm{~s}$. Further, in the drain current characterization the maximum drain current level of the P3CPT transistors is around two orders of magnitude higher $(\sim 100 \mu \mathrm{A})$ than the steady-state drain current levels for the other two polymers, i.e. in agreement with the results from the I-V characterizations. Noteworthy, the gate current level of the P3CPT transistors is more than one order of magnitude higher than for the $\mathrm{P} 3 \mathrm{HT}$ - and $\mathrm{P} 3 \mathrm{HT}-\mathrm{COOH} 15$ transistors. When integrating the gate currents to extract the total amount of charge stored in $C_{i}$ needed to modulate the transistors we record $380 \mu \mathrm{C} / \mathrm{cm}^{2}$ (P3CPT), $21 \mu \mathrm{C} / \mathrm{cm}^{2}$ (P3HT) and $97 \mu \mathrm{C} / \mathrm{cm}^{2}$ (P3HT-COOH15). As the ion density of a Helmholtz layer is limited to somewhere on the order of $10^{15} \mathrm{ions} / \mathrm{cm}^{2}$ the maximum capacitance in a Helmholtz layer should be limited to $\sim 100 \mu \mathrm{C} / \mathrm{cm}^{2}$. This value corresponds fairly well to what we measure for P3HT and P3HT-COOH15, but for P3CPT the charge storage level is far above this fundamental limit. If we assume that the major part of the voltage drop occurs over this single Helmholtz layer we can estimate the corresponding capacitance values; $75 \mu \mathrm{F} / \mathrm{cm}^{2}$ (P3CPT), $4 \mu \mathrm{F} / \mathrm{cm}^{2}$ (P3HT) and $19 \mu \mathrm{F} / \mathrm{cm}^{2}$ (P3HT-COOH15). These values are in fairly good agreement with the measured values found from the impedance spectroscopy and C-V experiments (Figure 4 and 5).

\section{Discussion}

From I-V measurements performed on the transistors, including the three different polymers, we observe much larger on-state drain currents for the transistor including the P3СРT material as compared to the devices including $\mathrm{P} 3 \mathrm{HT}$ and $\mathrm{P} 3 \mathrm{HT}-\mathrm{COOH} 15$ as the semiconductor channel. Assuming a field-effect mode of operation we can make an estimate of the field effect 
mobility in the linear regime $\mu_{\text {lin }}$ using the data from the I-V curves. We assume that the maximum capacitance per unit area is $C_{i} \leq 100 \mu \mathrm{F} / \mathrm{cm}^{2}$ (typical upper limit for Helmholtz layer capacitance).

$$
\mu_{\text {lin }}=L /\left(W C_{i} V_{D S}\right) * \partial I_{D} / \partial V_{G S}
$$

Then, the estimated $\mu_{\text {lin }}$ are $0.3 * 10^{-3} \mathrm{~cm}^{2} / \mathrm{Vs}(\mathrm{P} 3 \mathrm{HT}), 0.7 * 10^{-3} \mathrm{~cm}^{2} / \mathrm{Vs}(\mathrm{P} 3 \mathrm{HT}-\mathrm{COOH} 15)$ and $30 * 10^{-3} \mathrm{~cm}^{2} / \mathrm{Vs}$ (P3CPT). Using a polar gate insulator in OFETs is known to typically yield a relatively lower field effect mobility, than otherwise attained using for instance a dielectric insulator, for disordered OSCS [36]. When comparing our extracted mobility values to similar devices, with undoped OSCs, that are gated through a polar insulator or via an electrolyte [11, 26, 36-39] we find that the values extracted for $\mathrm{P} 3 \mathrm{HT}$ and $\mathrm{P} 3 \mathrm{HT}-\mathrm{COOH} 15$ seem to agree to prior reported data. The relatively much higher mobility value extracted for P3CPT leads us to suspect that there might be a different operation mechanism for P3CPT-OTFTs than for the other two polymer OSCs.

If the OTFTs, including the three different OSCs, are operating through field-effect modulation, the on-to-off and the off-to-on switching time should agree with the time period it takes to establish the Helmholtz layer [40] or the time it takes to establish a conducting channel. Using deionized water as the gate medium we expect that the drain current transient characteristics should be limited to around $0.1 \mathrm{~s}[26,41-42]$. Such drain current transient behaviour seems to be valid, more or less, for the case of P3HT and P3HT-COOH15 where both the on-to-off and off-to-on switch times are found to be less than $1 \mathrm{~s}$. The switching speed for the P3CPT-based transistors exhibit a much slower transient characteristics; both the on-to-off and off-to-on switch times are 1-2 orders of magnitude slower as compared to the other OTFTs. For transistors based on P3HT and P3HT-COOH15 the transient characteristics suggest that the operational mechanism is governed by the generation of a Helmholtz layer. For the P3CPT transistor the mode of operation rely on a much slower 
mechanism. In Figure 4, the establishment of the Helmholtz layer can be seen at frequencies of a few $100 \mathrm{~Hz}$ and below, which agrees well with the speed of operation for the EGOFETs. In the case of P3CPT the capacitance keeps increasing with decreasing frequencies below this point, indicating some secondary mechanism for build up of charges. Even at frequencies of down to $0.1 \mathrm{~Hz}$ the capacitance for the system with P3CPT does not seem to have reached a maximum. This agrees well with the speed of the OECTs. A possible mechanism is that protons migrate to the electrolyte and the carboxylic ions neutralize the positive doping charge carried by the polythiophene chains.

The current running through the gate while the transistors are switched from on to off was recorded. The amount of charges polarized and stored at the semiconductor-water interface can be integrated. If the $\mathrm{OH}^{-}$ions in the water sample are unable to penetrate into the semiconductor, a stable and dense Helmholtz layer is formed and would then constitute a limit to how much charge that can be stored. The amount of charge possible to store in a Helmholtz layer is typically limited to $\sim 100 \mu \mathrm{C} / \mathrm{cm}^{2}$. For a pure field-effect mode of operation the stored charges at the watersemiconductor interface should be $\leq 100 \mu \mathrm{C} / \mathrm{cm}^{2}$. P3HT $\left(21 \mu \mathrm{C} / \mathrm{cm}^{2}\right)$ and P3HT-COOH15 $\left(97 \mu \mathrm{C} / \mathrm{cm}^{2}\right)$ both fulfil this criterion, but P3CPT $\left(380 \mu \mathrm{C} / \mathrm{cm}^{2}\right)$ exhibit a much larger value. This leads us to suspect that charges are stored, not only along a planar interface, but rather inside the bulk of the semiconductor.

From the results of the impedance measurements it becomes clear that the capacitance value of P3CPT capacitors, when approaching lower frequencies, is much higher than expected for a compact Helmholtz layer. Assuming a smooth surface and high surface charge density of the semiconductor film the capacitance value should be limited to $\sim 20 \mu \mathrm{F} / \mathrm{cm}^{2}$. Towards lower frequencies the P3CPT capacitor exhibit a high capacitance value suggesting charge storage capacity beyond what is possible to store across a Helmholtz layer; yet another indication of bulk charge doping. 
The effect of the applied DC voltage on the capacitance value can reveal information regarding electronic and ionic charge injection and polarisation into the semiconductor. Charge injection into the semiconductor occurs at a voltage ranging from 0.8-1.2 V. For P3HT and P3HT$\mathrm{COOH} 15$ the capacitance value saturates at $3-4 \mu \mathrm{F} / \mathrm{cm}^{2}$. No saturation is found for P3CPT capacitors as the voltage is decreased. In fact, the capacitance reaches $40 \mu \mathrm{F} / \mathrm{cm}^{2}$ already at $-0.2 \mathrm{~V}$. As the voltage is further reduced the capacitance reaches $70 \mu \mathrm{F} / \mathrm{cm}^{2}$ at $-0.6 \mathrm{~V}$ (data not shown). Such nonlinear charge storage characteristics indicate bulk doping via electrochemistry.

Finally it should be mentioned that, even though the characteristics of P3HT-COOH15 seem to be dominantly governed by a field effect mode of operation, i.e. not providing enough anion mobility into the semiconductor bulk to be dominated by an electrochemical mode of operation, some ion mobility within the bulk of the material should perhaps not be excluded. This would in turn render some actuation of the bulk electron transport through electrochemical doping possible. In that case however, the effect of such actuation is much smaller than the surface effect at the conditions presented in this work. In this study, we choose to apply pure water as the electrolyte, since it provides us with a well-defined electrolyte system, based on dissociated $\mathrm{OH}^{-}$and $\mathrm{H}_{3} \mathrm{O}^{+}$ions. We believe that our findings are of importance for various kinds of electrolyte systems, such as those of biological and chemical applications.

\section{Conclusions}

The transistor characteristics as well as the impedance measurements performed on EGOTFTs, and their capacitor equivalent, point to the conclusion that P3HT and P3HT-COOH15 might be well suited as the semiconductor channel in OFETs gated through aqueous electrolyte. For P3CPT this does not seem to be the case as it appears susceptible to ion penetration and/or ion mobility within the bulk of the material. OTFTs gated through aqueous electrolyte with P3CPT as the semiconductor will thus be dominated by an electrochemical mode of operation including bulk 
doping thus separating channel charge conduction away from the aqueous interface. Through the collection of electrochemical, electrical and spectroscopic characterization protocols presented in this paper we have been able to determine that $\mathrm{P} 3 \mathrm{HT}-\mathrm{COOH} 15$ is a semiconductor channel material that exhibit field effect mode of operation. It also includes a relatively large density of anchoring sites for receptor molecules. This material is then a promising generic candidate in sensor transistors where the sensor reaction and signal transduction are both confined to the semiconductor-aqueous interface.

\section{Acknowledgements}

The authors wish to thank the EU for the prime support of this project here reported (BioEGOFET FP7 Grant Agreement $n^{\circ} 248728$ ). Toss, Kergoat, Crispin and Berggren also wish to thank the Swedish Government (SFO-AFM), the Önnersjö Foundation (Holmen), Knut and Alice Wallenberg Foundation (Power Papers) and VINNOVA (PEA) for additional financial support. Suspène wish to thank the University Paris Diderot for a 4 months extension of a post-doc position.

\section{References}

[1] F. Buth, A. Donner, M. Sachsenhauser, M. Stutzmann, J.A. Garrido, Biofunctional Electrolyte-Gated Organic Field-Effect Transistors, Advanced Materials, 24 (2012) 4511-4517.

[2] M. Demelas, S. Lai, G. Casula, E. Scavetta, M. Barbaro, A. Bonfiglio, An organic, charge-modulated field effect transistor for DNA detection, Sensors and Actuators B: Chemical, 171-172 (2012) 198-203.

[3] S.K. Kanakamedala, H.T. Alshakhouri, M. Agarwal, M.A. DeCoster, A simple polymer based electrochemical transistor for micromolar glucose sensing, Sensors and Actuators B: Chemical, 157 (2011) 92-97.

[4] J. Liu, M. Agarwal, K. Varahramyan, Glucose sensor based on organic thin film transistor using glucose oxidase and conducting polymer, Sensors and Actuators B: Chemical, 135 (2008) 195-199. 
[5] D.J. Macaya, M. Nikolou, S. Takamatsu, J.T. Mabeck, R.M. Owens, G.G. Malliaras, Simple glucose sensors with micromolar sensitivity based on organic electrochemical transistors, Sensors and Actuators B: Chemical, 123 (2007) 374-378.

[6] F. Biscarini, P.A. Stoliar, P. Greco, E. Bystrenova, F. Valle, A. Lazar, B. Chelli, F. Zerbetto, D. de Leeuw, Sensing Biomolecules with Ultra-Thin Film Organic Field Effect Transistors, Biophysical Journal, 98 (2010) 658a.

[7] H. Tang, P. Lin, H.L.W. Chan, F. Yan, Highly sensitive dopamine biosensors based on organic electrochemical transistors, Biosensors and Bioelectronics, 26 (2011) 4559-4563.

[8] D. Khodagholy, J. Rivnay, M. Sessolo, M. Gurfinkel, P. Leleux, L.H. Jimison, E. Stavrinidou, T. Herve, S. Sanaur, R.M. Owens, High transconductance organic electrochemical transistors, Nature communications, 4 (2013).

[9] H.U. Khan, M.E. Roberts, O. Johnson, W. Knoll, Z. Bao, The effect of pH and DNA concentration on organic thin-film transistor biosensors, Organic Electronics, 13 (2012) 519-524.

[10] S.H. Kim, K. Hong, W. Xie, K.H. Lee, S. Zhang, T.P. Lodge, C.D. Frisbie, Electrolyte-Gated Transistors for Organic and Printed Electronics, Advanced Materials, 25 (2013) 1822-1846.

[11] A. Laiho, L. Herlogsson, R. Forchheimer, X. Crispin, M. Berggren, Controlling the dimensionality of charge transport in organic thin-film transistors, Proceedings of the National Academy of Sciences, 108 (2011) 15069-15073.

[12] S.M. Goetz, C.M. Erlen, H. Grothe, B. Wolf, P. Lugli, G. Scarpa, Organic field-effect transistors for biosensing applications, Organic Electronics, 10 (2009) 573-580.

[13] Y. Hanazato, M. Nakako, M. Maeda, S. Shiono, Glucose sensor based on a field-effect transistor with a photolithographically patterned glucose oxidase membrane, Analytica Chimica Acta, 193 (1987) 87-96.

[14] L. Jagannathan, V. Subramanian, DNA detection using organic thin film transistors: Optimization of DNA immobilization and sensor sensitivity, Biosensors and Bioelectronics, 25 (2009) 288-293.

[15] L. Kergoat, B. Piro, M. Berggren, M.-C. Pham, A. Yassar, G. Horowitz, DNA detection with a water-gated organic field-effect transistor, Organic

Electronics, 13 (2012) 1-6.

[16] J.-M. Kim, S.K. Jha, R. Chand, D.-H. Lee, Y.-S. Kim, DNA hybridization sensor based on pentacene thin film transistor, Biosensors and Bioelectronics, 26 (2011) 2264-2269. 
[17] Q. Zhang, V. Subramanian, DNA hybridization detection with organic thin film transistors: Toward fast and disposable DNA microarray chips, Biosensors and Bioelectronics, 22 (2007) 3182-3187.

[18] S. Tiwari, A.K. Singh, L. Joshi, P. Chakrabarti, W. Takashima, K. Kaneto, R. Prakash, Poly-3-hexylthiophene based organic field-effect transistor: Detection of low concentration of ammonia, Sensors and Actuators B: Chemical, 171-172 (2012) 962-968.

[19] F. Yan, S.M. Mok, J. Yu, H.L.W. Chan, M. Yang, Label-free DNA sensor based on organic thin film transistors, Biosensors and Bioelectronics, 24 (2009) 12411245.

[20] Q. Zhang, L. Jagannathan, V. Subramanian, Label-free low-cost disposable DNA hybridization detection systems using organic TFTs, Biosensors and Bioelectronics, 25 (2010) 972-977.

[21] M.C. Tanese, D. Fine, A. Dodabalapur, L. Torsi, Organic thin-film transistor sensors: Interface dependent and gate bias enhanced responses, Microelectronics Journal, 37 (2006) 837-840.

[22] P. Stoliar, E. Bystrenova, S.D. Quiroga, P. Annibale, M. Facchini, M.

Spijkman, S. Setayesh, D. de Leeuw, F. Biscarini, DNA adsorption measured with ultra-thin film organic field effect transistors, Biosensors and Bioelectronics, 24 (2009) 2935-2938.

[23] M.E. Roberts, S.C.B. Mannsfeld, R.M. Stoltenberg, Z. Bao, Flexible, plastic transistor-based chemical sensors, Organic Electronics, 10 (2009) 377-383. [24] M. Magliulo, A. Mallardi, M.Y. Mulla, S. Cotrone, B.R. Pistillo, P. Favia, I. Vikholm-Lundin, G. Palazzo, L. Torsi, Electrolyte-Gated Organic Field-Effect Transistor Sensors Based on Supported Biotinylated Phospholipid Bilayer, Advanced Materials, (2013) n/a-n/a.

[25] M. Zayats, M. Lahav, A.B. Kharitonov, I. Willner, Imprinting of specific molecular recognition sites in inorganic and organic thin layer membranes associated with ion-sensitive field-effect transistors, Tetrahedron, 58 (2002) 815-824.

[26] L. Kergoat, L. Herlogsson, D. Braga, B. Piro, M.-C. Pham, X. Crispin, M. Berggren, G. Horowitz, A Water-Gate Organic Field-Effect Transistor, Advanced Materials, 22 (2010) 2565-2569.

[27] C. Suspène, L. Miozzo, J. Choi, R. Gironda, B. Geffroy, D. Tondelier, Y. Bonnassieux, G. Horowitz, A. Yassar, Amphiphilic conjugated block copolymers for efficient bulk heterojunction solar cells, Journal of Materials Chemistry, 22 (2012) 4511-4518.

[28] C. Suspène, B. Piro, S. Reisberg, M.-C. Pham, H. Toss, M. Berggren, A. Yassar, G. Horowitz, Copolythiophene-based water-gated organic field-effect transistors for biosensing, J. Mater. Chem. B, (2013). 
[29] J. Hou, Z.a. Tan, Y. Yan, Y. He, C. Yang, Y. Li, Synthesis and Photovoltaic Properties of Two-Dimensional Conjugated Polythiophenes with

Bi(thienylenevinylene) Side Chains, Journal of the American Chemical Society, 128 (2006) 4911-4916.

[30] H.-J. Wang, L.-H. Chan, C.-P. Chen, S.-L. Lin, R.-H. Lee, R.-J. Jeng, Bulky sidechain density effect on the photophysical, electrochemical and photovoltaic properties of polythiophene derivatives, Polymer, 52 (2011) 326-338.

[31] W. Li, B.J. Worfolk, P. Li, T.C. Hauger, K.D. Harris, J.M. Buriak, Self-assembly of carboxylated polythiophene nanowires for improved bulk heterojunction morphology in polymer solar cells, Journal of Materials Chemistry, 22 (2012) 11354-11363.

[32] J. Hou, T.L. Chen, S. Zhang, L. Huo, S. Sista, Y. Yang, An Easy and Effective Method To Modulate Molecular Energy Level of Poly (3-alkylthiophene) for High-Voc Polymer Solar Cells, Macromolecules, 42 (2009) 9217.

[33] N.D. Lang, W. Kohn, Theory of Metal Surfaces: Work Function, Physical Review B, 3 (1971) 1215-1223.

[34] G. Beltramo, M. Giesen, H. Ibach, Anomalous Helmholtz-capacitance on stepped surfaces of silver and gold, Electrochimica Acta, 54 (2009) 4305-4311. [35] H. Sirringhaus, Device Physics of Solution-Processed Organic Field-Effect Transistors, Advanced Materials, 17 (2005) 2411-2425.

[36] J. Veres, S. Ogier, G. Lloyd, D. de Leeuw, Gate Insulators in Organic FieldEffect Transistors, Chemistry of Materials, 16 (2004) 4543-4555.

[37] X. Yu, C. Jeong Ho, L. Jiyoul, P.P. Ruden, C.D. Frisbie, Comparison of the Mobility-Carrier Density Relation in Polymer and Single-Crystal Organic

Transistors Employing Vacuum and Liquid Gate Dielectrics, Advanced Materials, 21 (2009) 2174-2179.

[38] V.I. Arkhipov, E.V. Emelianova, P. Heremans, H. Bässler, Analytic model of carrier mobility in doped disordered organic semiconductors, Physical Review B, 72 (2005) 235202.

[39] C. Tanase, E.J. Meijer, P.W.M. Blom, D.M. de Leeuw, Local charge carrier mobility in disordered organic field-effect transistors, Organic Electronics, 4 (2003) 33-37.

[40] J. Lee, M.J. Panzer, Y. He, T.P. Lodge, C.D. Frisbie, Ion Gel Gated Polymer Thin-Film Transistors, Journal of the American Chemical Society, 129 (2007) 4532-4533.

[41] F. Grasso, F. Musumeci, A. Triglia, Impedance spectroscopy of pure water in the $0.01 \mathrm{~Hz}$ to $100 \mathrm{kHz}$ range, II Nuovo Cimento D, 12 (1990) 1117-1130. [42] L. Herlogsson, Y.Y. Noh, N. Zhao, X. Crispin, H. Sirringhaus, M. Berggren, Downscaling of Organic Field-Effect Transistors with a Polyelectrolyte Gate Insulator, Advanced Materials, 20 (2008) 4708-4713. 



\section{Figure Captions}
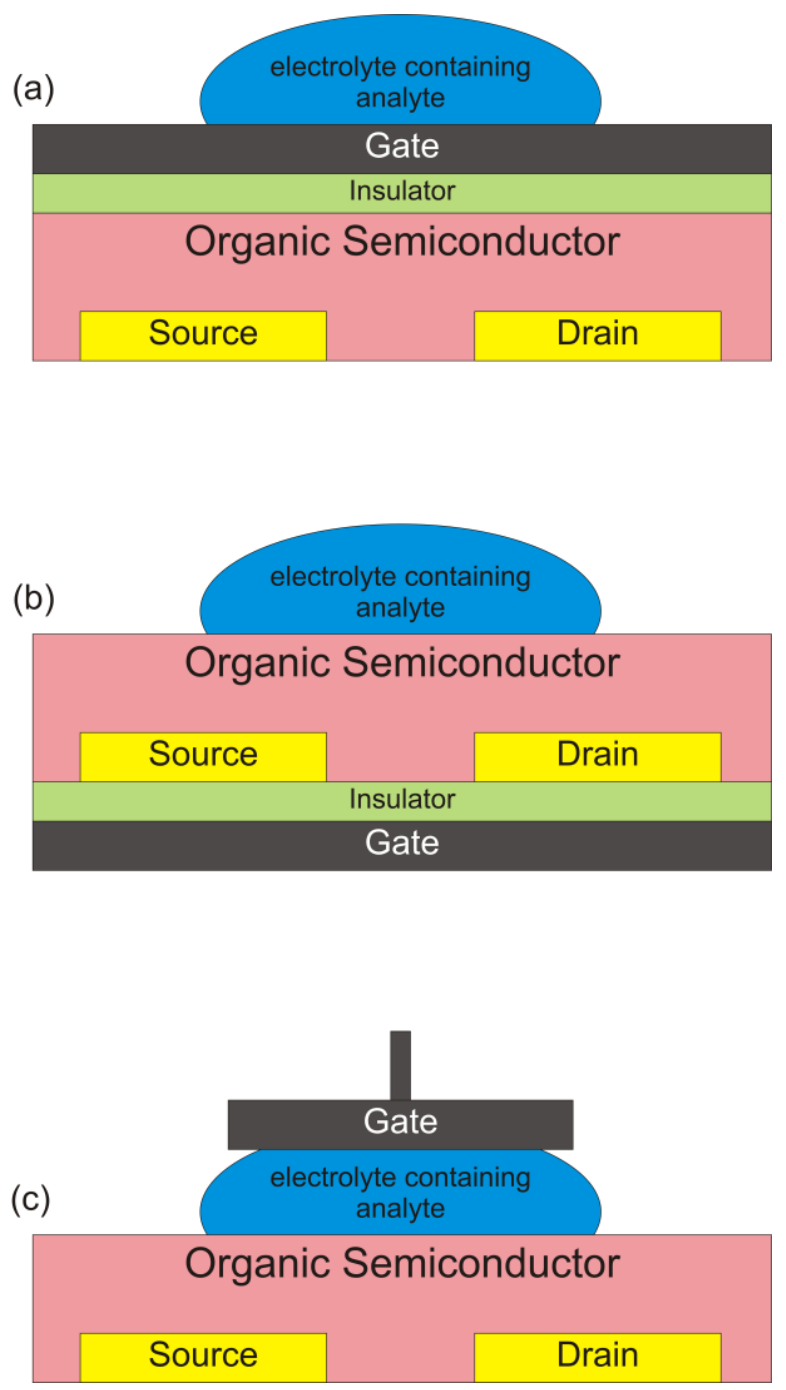

Figure 1 Three different possible set-ups for sensors with amplification of the sensing signal through OTFTs. The sensing elements are tentatively located; on the gate but separate from the OSC (a), on/in the OSC but separate from the gate (b), on either the gate or the OSC or in between the gate and the OSC (c). 
(a)

(b)

(c)

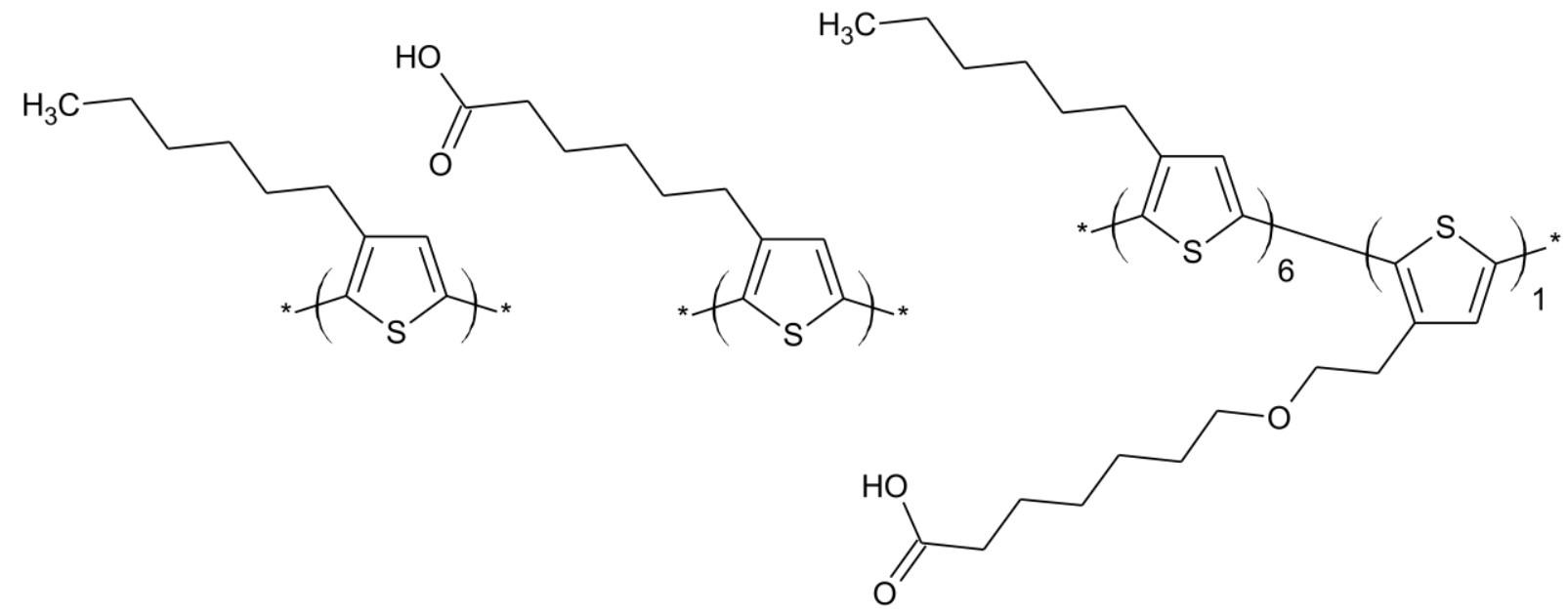

Figure 2 Chemical structure of P3HT (a), P3CPT (b) and P3HT-COOH15 (c).

(a)

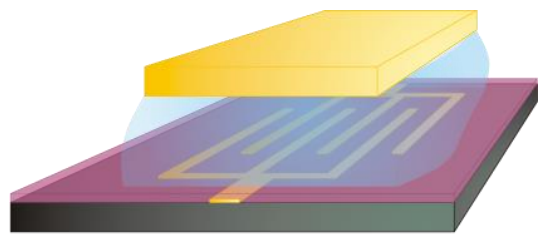

(b)

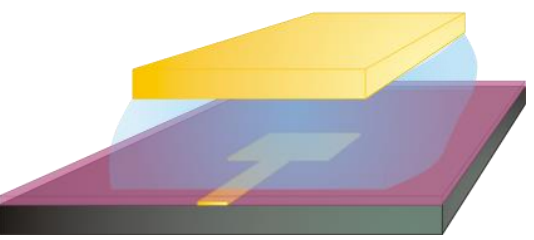

Figure 3 Schematic of the device architecture for the (a) water-gated EGOFET structures (interdigitated source/drain bottom electrodes; $L=2 \mu \mathrm{m}, W=2-10 \mathrm{~mm}$ ) and (b) capacitor (bottom capacitor plate $1 * 1 \mathrm{~mm}^{2}$ ). Top to bottom in both (a) and (b) the materials are Au (thick foil), $\mathrm{H}_{2} \mathrm{O}$ (few $\mu \mathrm{L}$ drop), OSC (thickness 10-20 nm), Au (50 nm thick bottom electrode)(Si/SiO substrate).

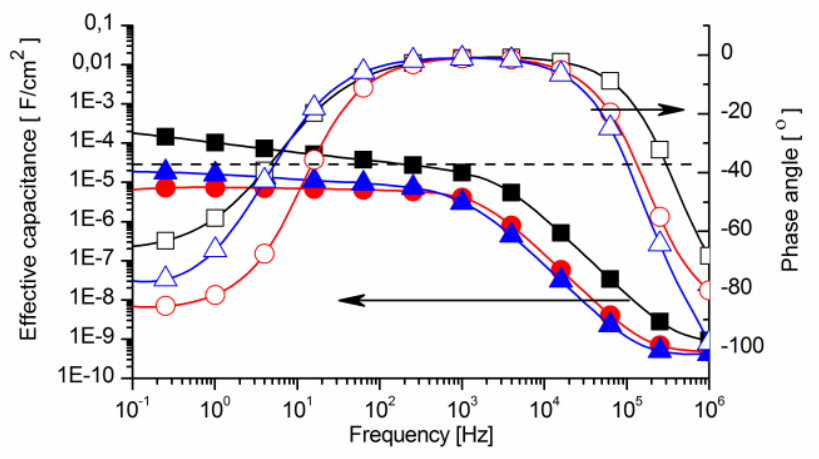

Figure 4 The effective capacitance $C_{\mathrm{i}}$ (filled symbols) and phase angle $\varphi$ (empty symbols) vs frequency for $\mathrm{P} 3 \mathrm{HT}$ (circles), P3HT-COOH15 (triangles) and P3CPT (squares). Dashed line denotes typical maximum effective capacitance value for a Au/water Helmholtz layer. $V_{A C}=0.1 \mathrm{~V}$ and $V_{D C}=0$ V. 


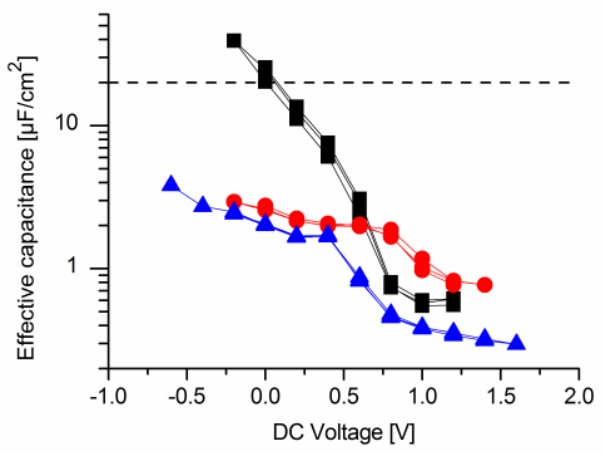

Figure 5 Effective capacitance vs DC voltage offset at constant frequency $100 \mathrm{~Hz} 0.1 \mathrm{~V} \mathrm{AC}$ signal for P3HT (circles), P3HT-COOH15 (triangles) and P3CPT (squares). Dashed line denotes typical maximum effective capacitance value for a $\mathrm{Au} /$ water Helmholtz layer. When reaching voltages of about $-0.6 \mathrm{~V}$ DC for P3HT the effective capacitance again starts to increase. This is suspected to be due to electrolysis.
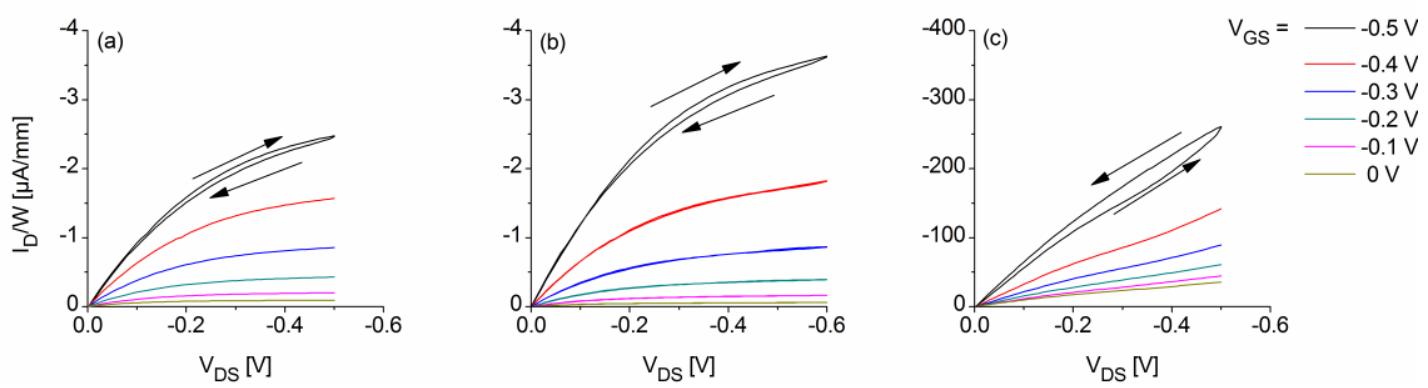

Figure 6 Typical transistor output characteristics curves for EGOFET devices, at different gate-source voltages $\mathrm{V}_{\mathrm{GS}}$, made using the three different organic semiconductors P3HT (a), P3HT-COOH15 (b) and P3CPT (c). Reverse sweep included for $V_{G S}=-0.5 \mathrm{~V}$. Arrows indicate sweep direction.
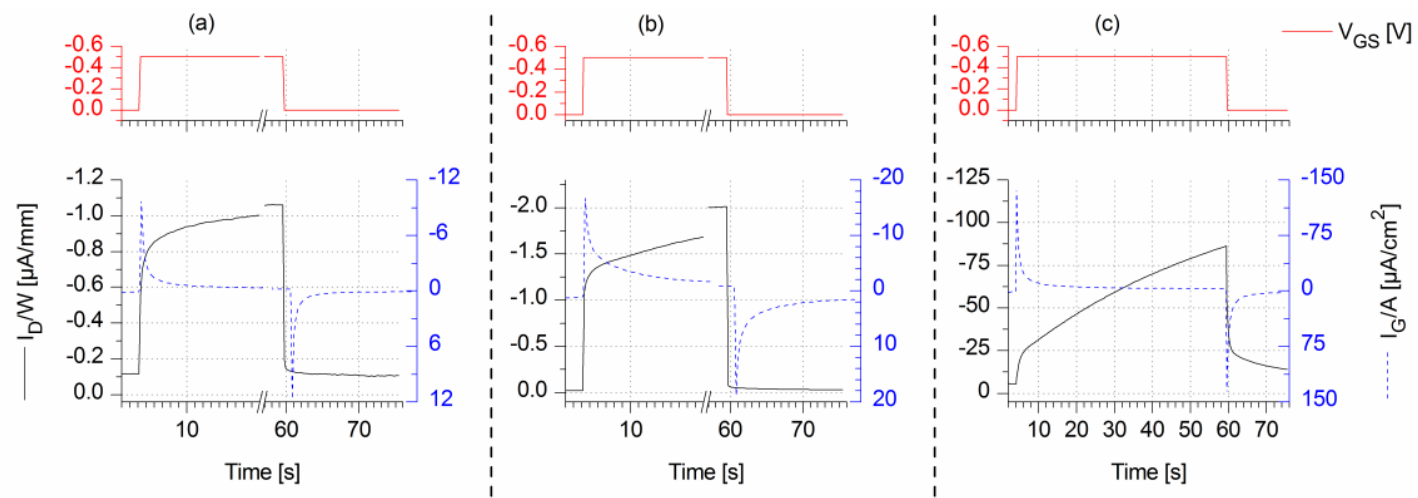

Figure 7 Transient behavior of transistors with the three different organic semiconductors. Drain current $\left(I_{D}\right)$ per transistor channel width $(W)$ and Gate current $\left(I_{G}\right)$ per channel + source electrode area $(A)$ are presented for more relevant comparison. For P3HT (a) and P3HT-COOH15 (b) there is a large, fast (on the order of $<1 \mathrm{~s}$ ) response in Drain current when the Gate-Source voltage is switched on after which the change is relatively small even over long periods of time (on the order of $60 \mathrm{~s}$ ). For P3CPT (c) the initial fast response is relatively small compared to the slow change which occurs over 
the long period of time. Note that the x-axes in (a) and (b) are cut to make the transient behavior in the fast switching more visible. 